\title{
Modeling on Energy Consumption of Cloud Computing Based on Data Center
}

\author{
Yu Yang ${ }^{1, \text { a }}$ Jiang Wei ${ }^{2, \text { a }}$ Guan Wei, a Li Ping $^{1 \text {, a }}$ Zhou Yongmin ${ }^{1, \text { a }}$ \\ ${ }^{1}$ China Academy of Engineering Physics, P.O.Box 919-71,Mianyang 621900,Sichuan,China \\ ${ }^{2}$ University of Electronic Science and Technology of China,Mianyang 621900,Sichuan,China \\ afresh1985@qq.com
}

Key Words: cloud data, energy consumption, model, algorithm

\begin{abstract}
: this paper puts forward the monitoring and measurement methods on energy consumption of virtual machine in the cloud data center, establishes energy consumption model of virtual machine system and virtual machine migration. The usual migration method of virtual machine uses heuristic algorithm to allocate virtual machine, its solution result is easily to be got into locally optimal solution, and this paper gives migration algorithm of virtual machine based on genetic algorithm on the basis of making research on genetic algorithm, it makes improvement on the target function in genetic algorithm, making application number of target node and migration time minimum on condition of meeting protocol of service class, so that it can realize energy conservation in data center.
\end{abstract}

\section{Introduction}

With the popularization and quick development ${ }^{[1,2,3]}$ of computer technology, the task request reaches in cloud computing platform becomes to be diversified. In order to meet task demand of different kinds, the calculation node in cloud data center constitutes hardware platform of cloud calculation has to keep open state for long time and wait for arrival of task, which will cause low application and high waste of cloud data center on energy consumption. Energy consumption model can be said to be one of the most important parts in cloud data center, when cloud data center is continually operating, we should make deep comprehension on users and administrators of cloud calculation, know their application way, so that make corresponding solution measures, so that it can reach target of optimization and energy conservation. At present, many servers of cloud data have the self-detection ability, they can measure some data components, but this single physical detection ability is obviously accords with future development idea of cloud data center, only quicker and effective energy conservation measures can make energy consumption modeling have cleanness ${ }^{[5,6]}$.

This paper tries to establish reasonable and reliable energy consumption model for cloud data center from layer of cloud infrastructure, and it compares with effect of different sampling ways and mathematical techniques on energy consumption model. On algorithm and test, it uses research outcome of energy consumption model put forward by this paper to demonstrate effectiveness of energy consumption model state by this paper, and this energy consumption model can be also applied to other research work on it.

\section{System structure on energy management of cloud computing}

It uses virtualized technology as core and fully considers resources application and load characteristics under cloud calculation environment, it makes effective monitoring management and optimization on energy consumption of cloud calculation, reduce total energy consumption of virtual cluster. It also provides calculation resources server on IT service, it can be indicated as DC = $\{\mathrm{H} 1, \mathrm{H} 2, \ldots, \mathrm{HN}\}$. It indicates calculation node, which is the physical server provides calculation ability, data center in distributed calculation, the node is divided into calculation node and storage node, data and file storage are in the storage node of NFS. The physical master is isostructural, it has the same calculation resources capacity(CPU, memory, disc), as the parasitifer, physical master 
opertes isomeric virtual machine, it is provided by Vmware or XEN. Virtual machine is isomeric, bit it is not random isomerism, it has certain virtual machine category defined in advance VC(vm - class) $=\{\mathrm{VCi},-, \mathrm{VCs}]$, virtual machine of every category is isostructural, its resources occupancy is (resource $)=\{\mathrm{R} 1, \ldots, \mathrm{RS}\}$. In order to record and describe state of current cluster, it adopts matrix to describe state in virtual cluster, the formula 1 is indicated as follows:

$$
C_{k}=\left[\begin{array}{lll}
\left(R_{1}, 1\right) & \left(R_{2}, 1\right) & \left(R_{3}, 1\right) \\
\left(R_{2}, 2\right) & \left(R_{3}, 2\right) & (0,2) \\
(0.3) & (0.3) & (0.3)
\end{array}\right]
$$

One self-management system structure EE Cloud used for energy conservation faced with virtual cluster of cloud computing, its overall structure is indicated by the following diagram 3-1. EECloud makes real-time monitoring on operation state of cluster and online energy consumption analysis by concentrated management, it also makes corresponding scheduling strategy by monitoring data analysis, so that it reaches self-management. Energy consumption management of EECloud mainly includes real-time cluster monitor, data analysis model, central control manager and the virtual cloud computing cluster controlled by it.

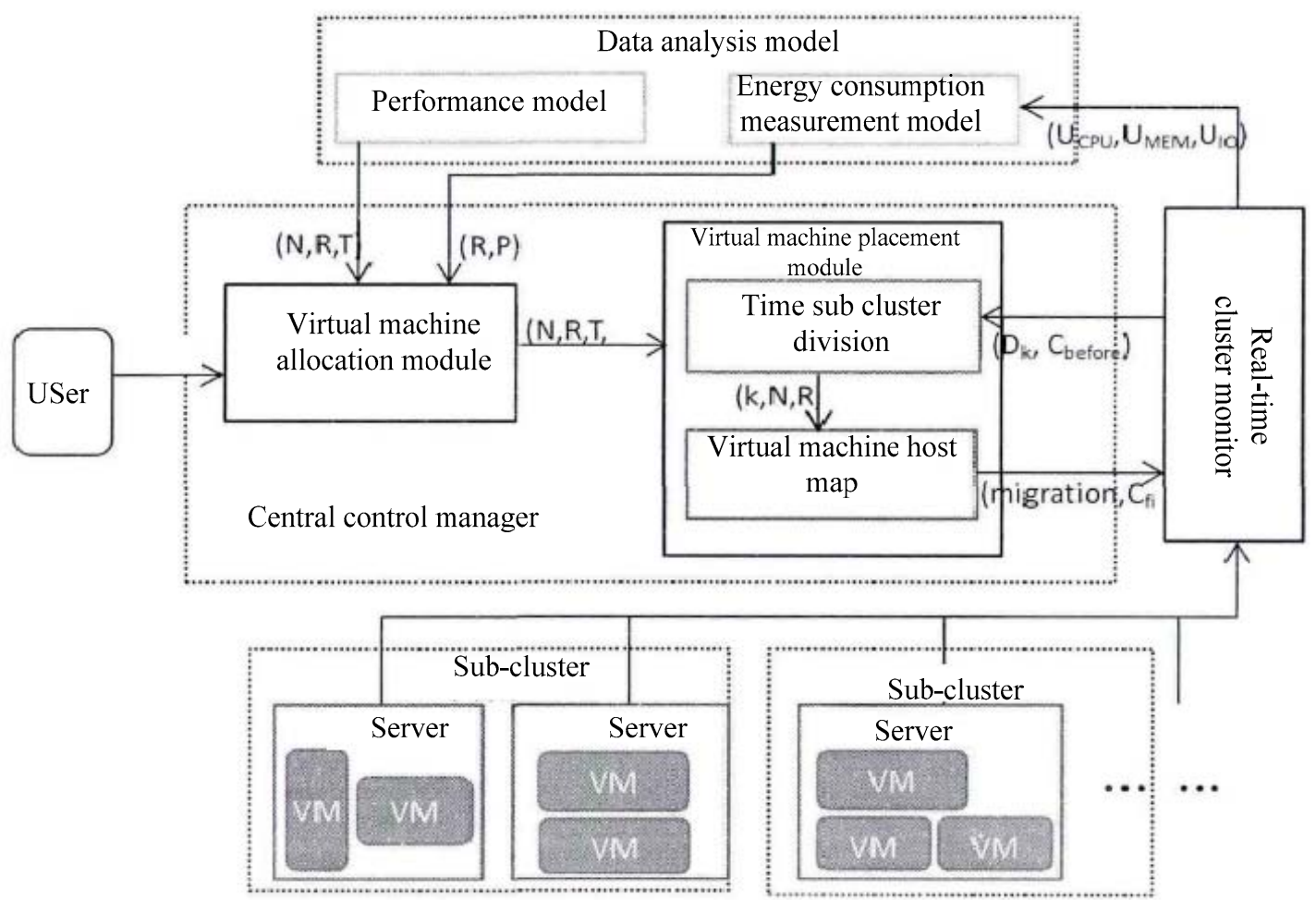

Diagram 1 3-1 Structure diagram of EECloud energy consumption

In order to overcome the above shortages and meet complicated scene including many entities in the simulation process, it develops one new event management structure above version of CloudSim 2.0, and diagram 2 is the basic design diagram of CloudSim. 


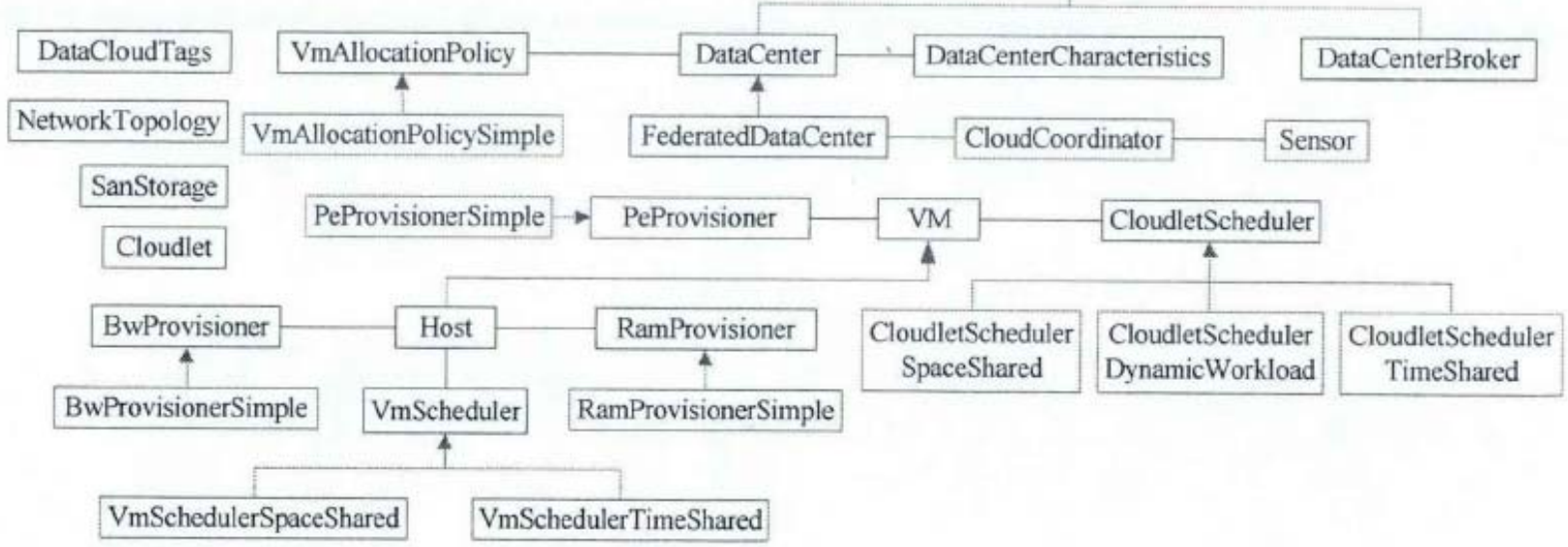

Diagram 2 Basic design diagram of CloudSim

\section{Energy consumption analysis and modeling in cloud data center}

Establish one energy consumption model; establish relations between availability (such as CPU) of certain kind of resources and energy consumption of overall system. As for one designated task, process time and availability information of processor are enough to evaluate the energy consumption of this task. Through calculation on energy consumption of every resource, it indirectly concludes energy consumption of CPU. In the given time period, as for one resources ri, processor availability Ui can be defined as follows:

$$
U_{i}=\sum_{j=1}^{n} u_{i, j}
$$

Of which, $\mathrm{n}$ indicates the total number of task in this time period, $\mathrm{u}$ ij indicates the utilization rate of task $\mathrm{t} j$ for resources ri . The calculation formula of $\mathrm{Uij}$ is as follows:

$$
u_{i, j}=\frac{i_{j}}{m_{j}} \times 100 \%
$$

$\mathrm{i}$ indicates the number of task $\mathrm{t} \mathrm{j}$ to use rsources, $\mathrm{m} \mathrm{j}$ indicates the total utilization amount on resources by task $\mathrm{t} j$. The energy consumption Ei of resources $\mathrm{r} i$ in the given time period can be defined as follows: of which, pmax indicates the energy consumption under the maximum load(or the energy consumption of processor has utilization rate of $100 \%$ ), p min indicates the minimum energy consumption under the minimum load/(or the energy consumption of processor has utilization rate of $1 \%)$, pmax and $\mathrm{p}$ min can be obtained by test.

$$
E=\left(p_{\max }-p_{\min }\right) \times U_{i}+p_{\min }
$$

The missing amount is easy to be obtained in the processor. Through these indexes, energy consumption model on memory energy can be indicated by formula (5):

$$
E_{m}(T)=\alpha_{m} N_{L}(T)+\gamma_{m}
$$

Of whicn, Em( T ) indicates the total energy consumption of memory in tine $\mathrm{T}, N_{L}(T)$ indicates the missing amount in the last layers of Cache in time T, $\alpha_{m}$ and $\gamma_{m}$ indicates parameter of linear model.

It uses parameter and data observed by management procedure to make modeling. However, management procedure can only see the readable and written Byte, as well as time of reading and writing these data. Therefore, it can use parameter and data observed by these management procedures to establish energy consumption model of disc.

$$
E_{d}(T)=\alpha_{r} b_{r}+\alpha_{w} b_{w}+\gamma_{d}
$$


Of which, $E_{d}(T)$ indicates energy consumption of disc in time T, $b_{r}$ and $b_{w}$ indicate Byte number of reading and writing in time $\mathrm{T}$. Transfer of virtual machine involves resources such as source node, network transmission, target node etc. Because transfer process is very complicated, energy expenditure is difficult to make quantization, so it only considers energy consumption of source node and target node on establishing evaluation model. Usually, the amount of data in source transmission is the same with the amount received by target node. Therefore, it can establish energy consumption model of virtual machine in the transfer process, the network expenditure caused by energy consumption and transfer of virtual machine presents relations of linear increase:

$$
E_{\text {mig }}=\alpha V_{\text {mig }}+\beta
$$

Of which, Emig is the energy consumption in the transfer process, it uses Emig to indicate, Vmig indicates network flow, it uses $\mathrm{Mb}$ to indicate, $\alpha$ and $\beta$ are model parameter.

: Energy consumption of master of virtual machine is as follows:

$$
E_{\text {host }}=E_{\text {min }}+c \mu^{2}
$$

Of which, $\mathrm{E}$ host is energy consumption of master of virtual machine, $\mathrm{E}$ min indicates the minimum energy consumption under the minimum load(or energy consumption of processor has utilization rate of $1 \%$ ), $\mathrm{c}$ is model parameter, $\mu$ is resources utilization rate of master node.

The energy consumption model of virtual machine transfer is as follows:

$$
E=E_{\text {host }}+E_{\text {mig }}
$$

\section{Transfer algorithm of virtual machine based on genetic algorithm}

\section{Decoding of chromosome coding}

Could computing is one large resources land, which provides service for each kind of users on the internet, it needs to meet different requirements of different users, some users have requirements on real-time, some users need low fees. Therefore, QoS demand in this chapter considers time of task implementation and fees in key point. Suppose there are $\mathrm{k}$ target node in data center, its number is respectively $\{\mathrm{H} 1, \mathrm{H} 2, \ldots, \mathrm{Hn}\}$. There are $\mathrm{n}$ virtual machines need to transfer to these $\mathrm{k}$ nodes, it needs to be remarked that sometimes several virtual machines can be transferred to the same target node, so it has no need to use all these $\mathrm{k}$ target nodes. The number seriation of each virtual machine transfers to target node has composed chromosome coding of this problem.

As for the first transfer plan(one of the individuals in the group), it indicates to transfer the $1, \mathrm{n}-1$ virtual machines to $\mathrm{H} 4$ nods, virtual machine of the 2, 4, $\mathrm{n}$ transferred to $\mathrm{H} 2$ node, the third virtual machine transferred to $\mathrm{H} 1$ node, the virtual machine of $\mathrm{n}-2$ transferred to $\mathrm{H} 7$ node. The initial group can be generated by random sequencing having $\mathrm{H} 1, \mathrm{H} 2 \ldots \mathrm{Hn}$. And then is decoding of chromosome coding, and gets transfer condition of virtual machine in the $\mathrm{k}$ target node, and it generates many groups of transfer sequence of virtual machine by using node number. The decoding of the above-mentioned is as follows:

$$
W_{1}:\{3\}, W_{2}:\{2,4, n\}, W_{4}:\{1, n-1\}, W_{7}:\{n-2\}
$$

The sequence after decoding can make statistics on certain transfer plan uses how many target nodes:

$$
F_{1}(x)=\sum_{j=1}^{k} y_{j}, W_{j} \in W
$$

\section{Fitness function and its improvement}

This target function not only considers the minimum amount of using target node, but also guarantees target node meets protocol of service class. Fitness function uses the following formula:

$$
F(x)=\left\{\begin{array}{lc}
C_{\text {max }}-E & \mathrm{E}(\mathrm{x})<\mathrm{C}_{\text {max }} \\
0 & \text { else }
\end{array}\right.
$$




\section{Individual selection}

The MGA algorithm in this paper uses proportional selection to select operator. Proportional selection methods means selecting the individual inherited to the next population according to fitness of individual in the population, the selected probability and fitness present relations of direct proportion. Proportional selection is one kind of method randomly selected, it is also regarded as Roulette Wheel selection, the selection principle and Roulette Wheel operation in the gamble is the same.

Interlace and mutation operation

MGA algorithm uses fitness function to confirm interlace probability function and mutation probability function. Interlace probability function and mutation probability functions are as follows:

$$
\begin{aligned}
& P_{c}= \begin{cases}K_{1}\left(F_{\text {max }}-F\right) /\left(F_{\text {max }}-F \arg \right) & \mathrm{E}(\mathrm{x}) \geq \mathrm{F}_{\text {arg }} \\
K_{2} & \text { else }\end{cases} \\
& P_{m}= \begin{cases}K_{3}\left(F_{\text {max }}-F\right) /\left(F_{\text {max }}-F \arg \right) & \mathrm{E}(\mathrm{x}) \geq \mathrm{F}_{\text {arg }} \\
K_{4} & \text { else }\end{cases}
\end{aligned}
$$

Fmax is the maximum fitness value in group, Farg indicates the average fitness value of each generation group, $F^{\prime}$ is the larger fitness value between 2 individuals on interlace, $F$ is the fitness value of planning mutation. It uses formula(4-9) to obtain fitness calculation Pc 、 Pm. Generation of one piece of chromosome is not randomly generated, while it transfers the first set of virtual machine to the first target node, it calculates how much is the remaining resources utilization rate of target node, it makes comparison between remaining resources utilization rate and that of other virtual machines, transfers the most adjacent virtual machine to this target node. If resources utilization rate of all the virtual machines are bigger than that of remaining resources utilization rate of this target node.

Analysis on simulated test

This paper uses Cloudsim to simulate transfer of virtual machine in cloud data center. It simulates one data center in the test, it compares with application number of target node and transfer time of virtual machine in the real-time transfer process by real-timely monitoring physical node, CPU utilization rate of virtual machine and transmission information in the transfer process. It also uses energy consumption model to make measurement, evaluation and analysis on energy consumption in the transfer process, it makes simulation for many times on ST, DT, and MGA strategy by giving different threshold.

In the simulation process on transfer algorithm of virtual machine, it needs to allocate environment and setting parameter. Table 1 gives the realized parameter setting table in the simulated data center, table 2 gives the main parameters of MGA algorithm.

\begin{tabular}{|c|c|c|c|c|c|c|}
\hline $\begin{array}{c}\text { Test } \\
\text { parameter }\end{array}$ & $\begin{array}{l}\text { Number } \\
\text { of } \\
\text { physical } \\
\text { master }\end{array}$ & $\begin{array}{l}\text { Calculation ability } \\
\text { of master CPU }\end{array}$ & $\begin{array}{c}\text { Master } \\
\text { memory/MB }\end{array}$ & $\begin{array}{c}\text { Number } \\
\text { of } \\
\text { virtual } \\
\text { machine }\end{array}$ & $\begin{array}{c}\text { Calculation ability of } \\
\text { CPU }\end{array}$ & $\begin{array}{c}\text { Times of } \\
\text { implemented } \\
\text { task }\end{array}$ \\
\hline Value & 200 & $\{1000,2000,3000\}$ & 1024 & 1000 & $\{250,500,750,1000\}$ & 100 \\
\hline \multicolumn{7}{|c|}{ Table 2 Main parameter of algorithm } \\
\hline project & \multicolumn{2}{|c|}{$\begin{array}{l}\text { Population } \\
\text { scale }\end{array}$} & $\mathrm{K}_{1}$ & $\mathrm{~K}_{2}$ & \multicolumn{2}{|c|}{$\mathrm{K}_{4}$} \\
\hline value & & 60 & 0.35 & 0.87 & 0.093 & 056 \\
\hline
\end{tabular}

Table 1 Parameter setting in data center

Through simulation test, test result of virtual machine transfer algorithm based on genetic algorithm is indicated by diagram 3 , 

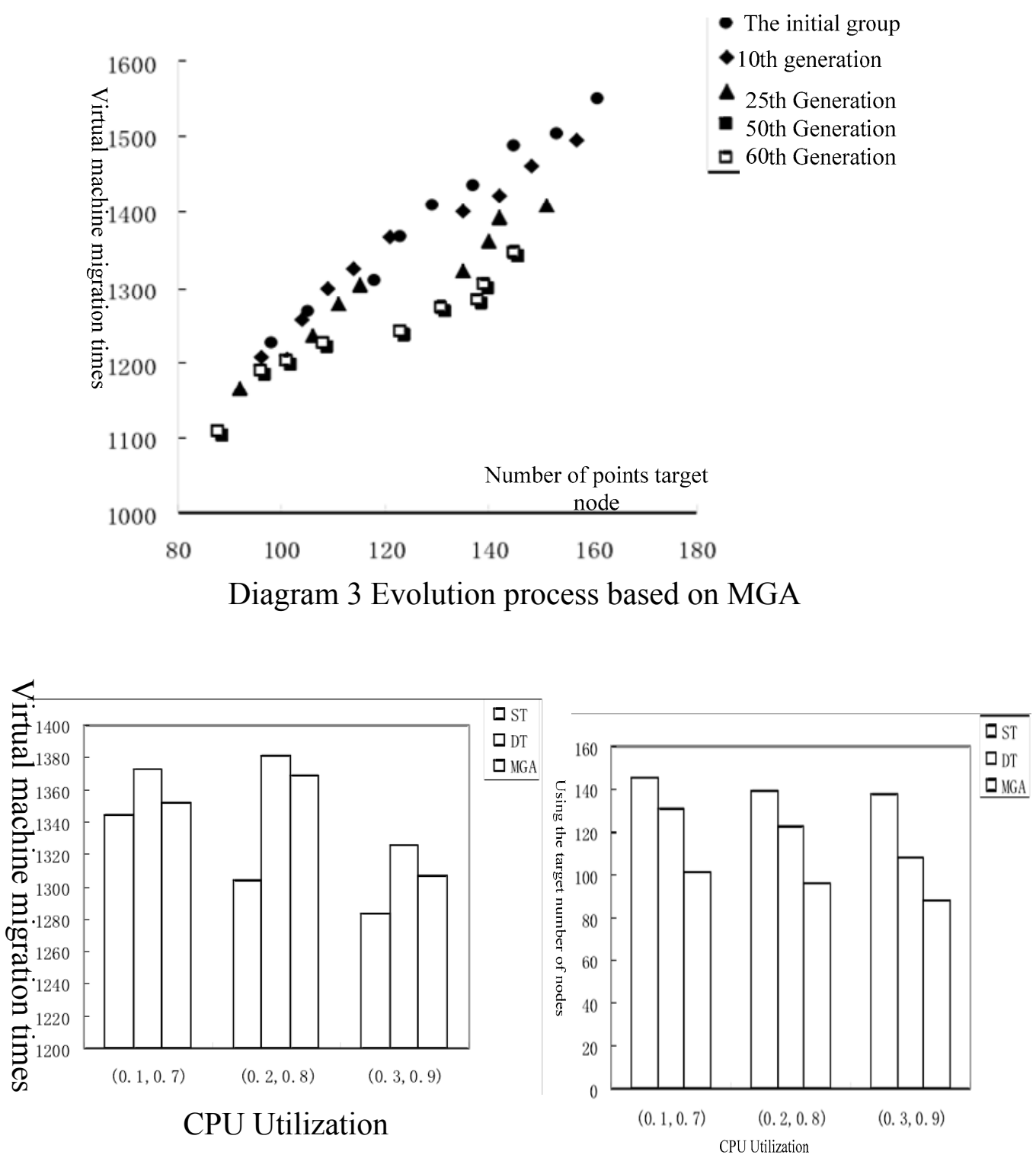

Diagram 4 Transfer time of virtual machine under different strategy Diagram 5 Application number of target bode under different strategy

According to the comparison results on strategy of this paper strategy, ST, DT strategy in aspects of transfer time of virtual machine, application number of target node, SLA destroy rate etc. DT and MGA set one more threshold on the basis of ST strategy, so there are more transfer times, but the number of using target node is less. Compared with DT strategy, MGA can effectively make thorough search on space of target node, so it can use fewer target nodes to meet target of service class, but contravention rate of target of service class is relatively higher and transfer time of virtual machine is relatively little.

By making real-time monitoring on CPU utilization rate of physical master and information transmission amount in the transfer process, it uses virtual machine and energy consumption model of virtual machine transfer to make measurement on energy consumption in the transfer process. Table 3 gives the comparison results of method in this paper, ST method, DT method in aspects of energy consumption, transfer time of virtual machine and SLA destroy rate etc. 
Table 3 Energy consumption=

\begin{tabular}{llll}
\hline Test method & $\begin{array}{l}\text { Energy } \\
\text { consumption } /(\mathrm{kWh})\end{array}$ & $\begin{array}{l}\text { migration time of } \\
\text { virtual machine }\end{array}$ & SLA \\
\hline ST(0.7) & 9.55 & 1345 & 10.16 \\
ST(0.8) & 9.23 & 1307 & 10.37 \\
ST(0.9) & 9.03 & 1291 & 10.49 \\
DT(0.1-0.7) & 7.75 & 1372 & 11.61 \\
DT(0.2-0.8) & 7.56 & 1378 & 11.88 \\
DT(0.3-0.9) & 7.34 & 1329 & 12.01 \\
MGA(0.1-0.7) & 7.14 & 1349 & 12.65 \\
MGA(0.2-0.8) & 6.82 & 1368 & 12.83 \\
MGA(0.3-0.9) & 6.69 & 1311 & 12.93 \\
\hline
\end{tabular}

On effect of energy conservation, MGA method has obvious enhancement compared with ST method and DT method. On condition of the same trigger threshold value, compared with DT, energy consumption and migration time of virtual machine of MGA all have reduced, this is because MGA makes optimization in aspects of choice of virtual machine, matching between migration virtual machine and target master etc on the basis of DT, which enables migration of virtual machine have much more efficiency.

\section{Summary}

With the increase of threshold for 3 kind of methods such as ST, DT and MGA, energy consumption in cloud data reduces, while destroy rate of SLA increases, this indicates that under certain condition, in order to realize energy conservation, it realizes energy conservation at the cost of reducing QoS of users. By comparing with application number of target node under migration strategy for task in cloud center as well as migration time of virtual machine and energy consumption condition, virtual machine algorithm based on genetic algorithm not only increases search speed but also reduces migration time and application number of physical node, enhances resources availability in cloud data center and reduces energy consumption.

\section{References}

[1] Chen Kang, Zheng Weimin. Cloud Computing: System Example and Research State. Software Journal, 2009, 20(5), p71-82.

[2] Jia Zhiping, Ju Lei etc. Scheduling and Optimization Method on Energy Conservation of Parallel Task Towards isostructural Cluster System. Computer Journal, 2012, 35(3): p59-66.

[3] Tian Guozhong, Xiao Chuangbo, Xu Zhusheng etc. Mixed Scheduling Strategy of Multi-DAG Work Flow under Isomeric Distributed Environment. Software Journal, 2012, 23(10), p 2720-2734..

[4] Nathuji R, Schwan K, Somani A, Joshi Y.VPM tokens: Virtual machine-aware power Budgeting in datacenters [J].Cluster Computing, 2009, 12(2):189-203.

[5] Snowdon D C, Le Sueur E, Petters S M, et al. Koala: A platform for OS-level power management $[\mathrm{C}] / /$ Proceedings of the 4 th ACM European conference on Computer systems. ACM, 2009, p289-302..

[6] Hyun-Jeong Jo,Jong-Gyu Hwang,et al.Formal requirements specification in safety-critical railway signaling system.TD-ASIA.2009.pp:1-4. . .

[7] Wang An. Research on Key Technology of Mobile Network Transmission towards Energy Conservation. Beijing University of Posts and Telecommunications, 2013.:228-233. . 
[8] GUO M Y.Green computing: connotation and tendency[J].Journal of Computing Engineering, 2010,36(10),p1-7.

[9] Song Jie, Li Tiantian, Yan Zhenxing etc. Energy Efficiency Model and Measurement Method under One Kind of Cloud Computing Environment. Software Journal, 2012,23(2). P200-214.

[10] Tan Yiming, Zeng Guosun, Wang Wei . Optimization and Management Method of Random Task in Cloud Calculation Platform. Software Journal 2012, 23(2), p266-278. 